\title{
Speedometer monitoring when driving with a speed warning system
}

\author{
Esko Lehtonen ${ }^{1 *}$, Neha Malhotra ${ }^{2,3}$, Nicola J. Starkey ${ }^{3}$ and Samuel G. Charlton ${ }^{3}$
}

\begin{abstract}
Aim: The study investigated how a speed warning system influence drivers' speedometer monitoring, and how drivers respond when they receive a speed warning. With a speed warning system, drivers may pay more attention to the exact speed, which would be expected to increase glances to the speedometer.

Methods: Nineteen participants drove in a driving simulator, either with or without a speed warning system implemented on a smartphone, which alerted them if they exceeded the set speed limit. Their glances to the speedometer and speed warning system's display were recorded with an eye tracker.

Results: Drivers sped less with the warning system. The system did not significantly change the speedometer monitoring. Speeding was preceded by fewer speedometer glances, indicating the speeding was linked to participants' momentary inattention to their speed control. After a warning, the frequency of speedometer glances increased. Interestingly, drivers often started to glance at the speedometer and release the accelerator a couple of seconds before a warning, suggesting that drivers learn to anticipate warnings. The results suggest that drivers do not only react to warnings, but they also actively try to avoid them. Understanding drivers' speed control with speed warning system may help to create adaptive ISA systems.
\end{abstract}

Keywords: Eye movements, Intelligent speed adaptation, In-vehicle information systems, Distraction, Anticipation

\section{Introduction}

Speeding increases crash risk and the consequences of crashes $[1,6]$. Drivers' speed choice is influenced by many factors, including the environment, the purpose of the trip, and personal preferences [3]. In many instances, however, the speeds people choose to drive are not safe, and not in agreement with the speeds other drivers choose $[1,3,6]$. Intelligent Speed Adaptation (ISA) systems are a promising technological solution to increase compliance with speed limits and promoting speed homogeneity $[2,24]$. ISA systems can function either by issuing a speed warning when the driver is exceeding the speed limit, or by directly controlling the speed of the vehicle [25]. Recently, the European Union proposed a regulation that all new passenger vehicles should be equipped with ISA by 2022 [8].

The current eye-tracking study was a part of a larger study $(n=104)$ investigating the effect of ISA system on

\footnotetext{
* Correspondence: esko.lehtonen@vtt.fi

${ }^{1} \mathrm{VTT}$ Technical Research Centre of Finland, Espoo, Finland

Full list of author information is available at the end of the article
}

driving performance in a simulator [23]. The study found that ISA leads to better speed compliance and did not impair lane keeping. Based on the eye-tracking results further analysed in the current study, the ISA system was evaluated not to be visually distractive, as drivers glanced at the ISA rather infrequently and briefly. However, the ISA system can still change the way drivers use the speedometer to regulate their speed, which is the focus of the current study.

The speedometer is mostly needed when the drivers are required to deliberately maintain the set speed limit. In fact, there are many studies which have shown that occluding a speedometer may not substantially change normal driving (e.g. $[15,22])$. The need to look at the speedometer arises mostly when drivers want to maintain a speed which is lower than the one they would drive if they could choose freely [22].

The optimal speed hypothesis [22] states that drivers prefer to choose a speed which minimizes their cognitive load in the current driving situation $[10,22]$. Such an optimal speed would be the one which matches the road and traffic environment, and the skills and preferences 
of the driver. Drivers may drive slower or faster than the optimal speed, if they have a reason to do so, but this requires cognitive effort and control [7]. From this perspective, a driver who has an optimal speed higher than the current speed limit, could momentary speed if he/ she has a lapse of cognitive control. Such a lapse of cognitive control would manifest as a decrease in speedometer glances.

In this study, we investigated drivers' speedometer monitoring (glances) when driving with or without a speed warning system. First, we wanted to investigate if the drivers start to use more the speedometer when driving with a speed warning system, as they try to maintain a lower speed they otherwise would. This could manifest itself as an increase overall speedometer glance rate, but possibly also as an increasing synchronization of speedometer glances and speed regulation action. There is a general pattern that gaze leads actions by $0-2 \mathrm{~s}$ : for example, before grasping and moving an object humans typically look at the object just before they reach or using it $[11,13$, 21]. In locomotion, gaze anticipates changes in direction and foot placements $[14,17,20]$. In driving, humans prefer to look before they steer [16]. Similarly, it could be expected that drivers would look at the speedometer before they press or release the accelerator pedal. However, this preference to look before doing is driven by the need to obtain information, that is, to reduce the uncertainty over the current state of the world. Similarly, speed warnings would be expected to trigger glances toward the speedometer, as the drivers need to adapt their speed.

Second, we were also interested if speed warnings were preceded by a period of decreased speedometer glance rate, indicating a momentary lapse of cognitive control over the speed before the speeding occurs. Speed warning could also change the typical gaze-leads-action synchronization. When the necessary information is already available without direct gaze, e.g. using peripheral vision (e.g. [16]) or via an auditory warning, there is not necessarily a need to glance at the speedometer before releasing the accelerator.

Third, drivers' speedometer glance rates in speed limit changes were also investigated. At speed limit changes, speed adaptation is similarly required. If we would detect an increase in the speedometer glances during the speed adjustment, we can interpret with larger confidence, that increase in speedometer glance rate as a response to a warning could be related to speed adaptation, and is not only due to a warning.

\section{Methods}

\subsection{Participants}

Nineteen volunteers (8 male) were recruited to complete a drive in the simulator equipped with a speed warning system while their eye movements were tracked. Participants had to be able to drive without glasses or contact lenses. The participants' average age was 27.53 years, $(\mathrm{SD}=6.32)$ and they had an average of 9.26 years of experience as licenced drivers $(\mathrm{SD}=6.74)$. They reported an average of $175.95 \mathrm{~km}$ driving per week $(\mathrm{SD}=170.05)$. This research complied with the tenets of the Declaration of Helsinki and was approved by the School of Psychology Research and Ethics Committee, University of Waikato (Ref 17/14).

\subsection{Simulator and the speed warning system}

The experiment took place in the University of Waikato fixed-base driving simulator which consisted of a complete automobile (2010 Toyota Prius plug-in) positioned in front of three angled projection surfaces. As has been described in Starkey et al. [23], the centre projection surface was located $2.32 \mathrm{~m}$ in front of the driver's eye position with two peripheral surfaces connected to the central surface at $52^{\circ}$ angles. This configuration produced a $178.2^{\circ}$ (horizontal) by $33.7^{\circ}$ (vertical) forward view of the simulated roadway from the driver's position. The vehicle model and the simulator screens were updated at least $100 \mathrm{~Hz}$. The simulator hardware and software was developed by the Transport Research Group. Its validity against on-road performance has been tested in several different kinds of experimental scenarios, including those with a focus on speed choice (e.g. $[4,5])$.

Four small infra-red LEDs were placed on the outside of the car windscreen to facilitate calibration and analysis of their eye movements. The simulated speed was displayed in digital form on the dashboard. The speed warning system was implemented as an application on a smartphone attached to the console to $12 \mathrm{~cm}$ to the left of the steering wheel. Visually, the display was located $10.6^{\circ}$ below, and $29.3^{\circ}$ to the left of the driver's forward line of sight.

A speed warning was triggered if the driver exceeded the speed limit by $4 \mathrm{~km} / \mathrm{h}$ for $3 \mathrm{~s}$. Speed warnings consisted of a large speed rondel (display size of $2.7 \mathrm{in}$., resolution of $1280 \times 720$ ) flashing and beeping until the participant reduced their speed to within $4 \mathrm{~km} / \mathrm{h}$ of the speed limit (Fig. 1). The speed warning app had two modes, passive and active. In the passive mode, the display automatically updated to show the new speed limit after entering a new speed zone. In the active mode, the participants were told to select the new speed limit when they entered a new speed zone (from the small speed limit signs along the bottom of the display screen). If the driver forgot to select the speed, it was automatically updated to the correct speed after $7.0 \mathrm{~s}$.

\subsection{Procedure}

Participants drove a simulated $26.4 \mathrm{~km}$ rural road. The drive had two $3 \mathrm{~km}$ baseline sections at the beginning and end of the drive $(80 \mathrm{~km} / \mathrm{h})$, and six sections with 60 


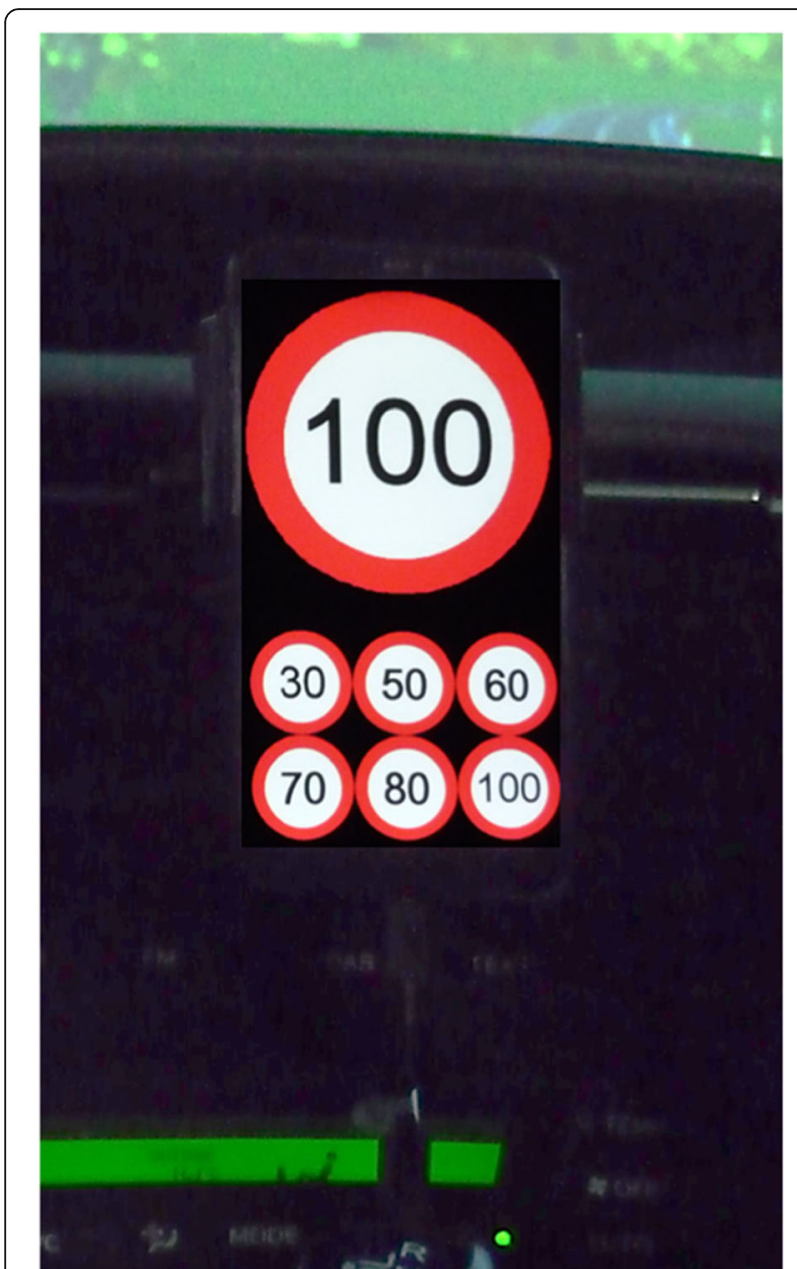

Fig. 1 The display of the speed app used in the study. Above, the current speed limit is indicated by a large rondel, which also flashed as a warning to drivers exceeding the speed limit. Using the smaller rondels below, the driver was able to set the current speed limit in the Active condition

$\mathrm{km} / \mathrm{h}, 80 \mathrm{~km} / \mathrm{h}$ and $100 \mathrm{~km} / \mathrm{h}$ speed zones, as well as one work zone with $30 \mathrm{~km} / \mathrm{h}$ (Fig. 2). It took approximately 20 mins to complete the drive. The speed warning system functioned throughout the route, except in the baseline sections (and for participants in the control condition).

The simulated road had some potential hazards, which required the driver to stay alert, including oncoming traffic, intersections with vehicles entering or exiting, roadworks and a one-way bridge and one overtaking situation, in which the driver could try to overtake a truck on a passing lane. If not, the truck pulled over on the shoulder to let the driver pass. Except for the truck, there was no other traffic in the driver's lane.

The participants were randomly assigned either to the control group (speed warning system was turned off), or to complete the drive with the system in either the active or passive mode. The final composition of the groups is shown in Table 1. Because there were no large differences between the active and passive mode regarding the speeding or speedometer monitoring (Supplementary Tables 1 and 2), the groups were combined for analysis.

\subsection{Eye-tracking}

Gaze was tracked with Tobii Pro Glasses 2, a binocular head-mounted eye-tracker which could be worn like glasses. The eye tracker was calibrated using the manufacturer's one-point calibration method, where the participant was asked to look at a marker placed on the windscreen wipers (the marker was removed before the drive). Calibration accuracy was tested qualitatively before and after a drive by asking the driver to look at designated points in the car (smartphone, speedometer, side and rear mirrors, four markers placed on the windscreen). Two participants, one from the active and one from the control group, were excluded from the analysis due to inadequate eye tracking quality.

Fixations and saccades were detected with Tobii Pro Lab 1.58 analysis software using IV-T algorithm with a velocity threshold of $100 \mathrm{deg} / \mathrm{s}$. Such a high threshold was recommended by the manufacturer to measure periods of foveal stabilization in a dynamic environment. That is, also periods of smooth pursuit and vestibular ocular reflexes will be counted as a fixation. The speed app and speedometer areas of interests were defined for each participant. A fixation was categorised as a speedometer or speed app fixation if most of the gaze data points were within the area of interest.

The aforementioned fixations were used and their frequency and durations were already reported in Starkey et al. [23]. Because the timing of glance onsets was critical for the present analysis, we further processed the data. Glances were defined to start from the beginning of a saccade followed by a fixation to the speedometer or speed app area of interests (AOIs). Consecutive saccades/fixations within the AOI were merged within the same glance.

\subsection{Analysis}

To measure if the drivers start to use more the speedometer when driving with a speed warning system, we calculated overall speedometer glance rates for each road section (for sections, see Fig. 2). Speedometer glance rates were calculated by dividing the number of glances by the duration of the section. For comparison, the speed app glance rates were calculated similarly. Average speeds and the proportion of time spent speeding were also calculate for each section.

Also, we wanted to understand how the speedometer glance rates in the simulator would compare to the speedometer glance rates in real-world driving. Because we were not able to find speedometer glance rates reported comparably from the literature, we calculated them ourselves using publicly available data from Lehtonen et al. [16]. Data were 


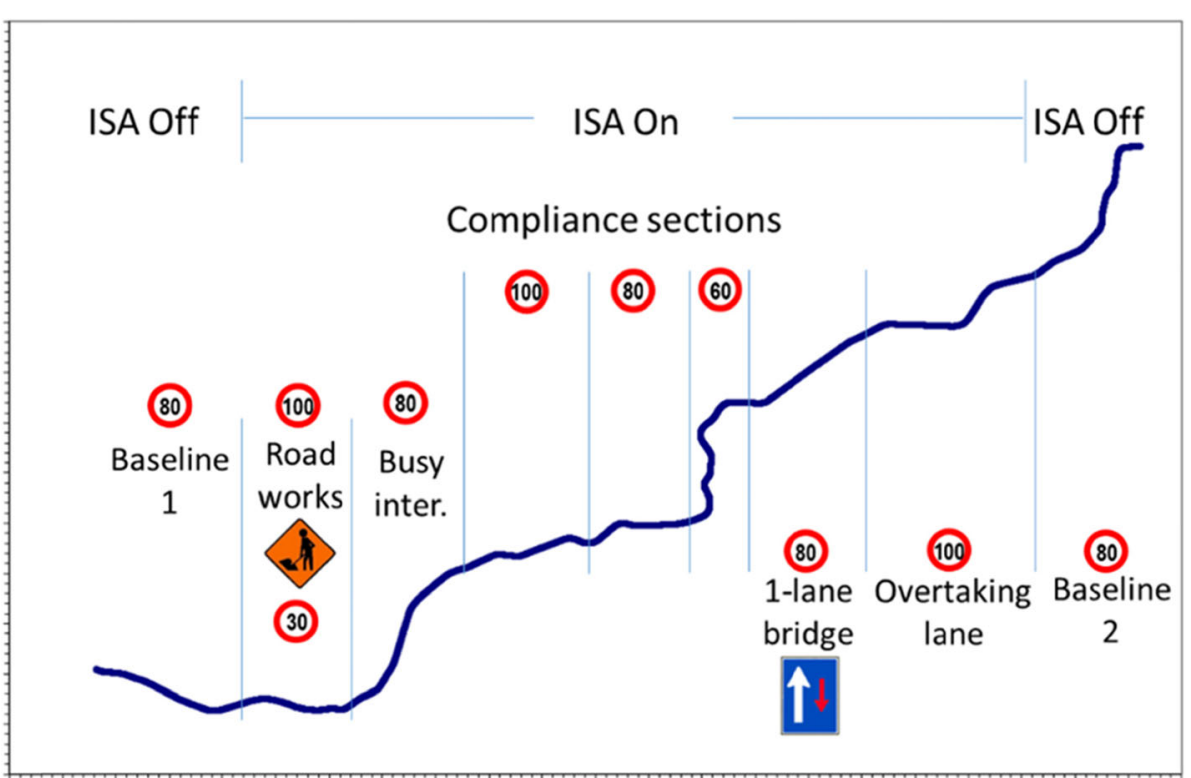

Fig. 2 Schematic map of the simulated road with speed limit sections and potential hazards

from an experiment, where drivers drove an instrumented car on a $2+2$ lane motorway while their eye movements were recorded. Data from 14 drivers during their control drives without a secondary task were used. During the control drives, they were asked to keep to the inside lane and drive at $90 \mathrm{~km} / \mathrm{h}$. Nine of the drivers had driven more than $30,000 \mathrm{~km}$ and held a license for more than 2 years, five less than that. Recording duration was $442-510 \mathrm{~s}$ for 12 drivers. Two drivers only had half of the control drives recorded (234 s and $235 \mathrm{~s}$ ). Speedometer glance rates were calculated by dividing the speedometer glance frequency by the duration of the recording.

To investigate if the speed app increased synchronization of the speedometer glances with the accelerator releases, glance data for the 30 segment before and after each accelerator release were extracted. Then the number of glances were calculated for each $1 \mathrm{~s}$ bin before and after a transition/warning. The corresponding speedometer glance rate was calculated by dividing the number of glances in each 1 $s$ bin by the number accelerator releases.

To investigate if speed warnings were preceded by a period of decreased speedometer glance rate, a similar analysis was performed. Data were extracted for the $30 \mathrm{~s}$ segment before and after each warning. Then the number of glances and the average speed were calculated for each $1 \mathrm{~s}$ bin before and after a warning. The corresponding speedometer glance rate was calculated by dividing the number of glances in each $1 \mathrm{~s}$ bin by the number of warnings/transitions encountered. For speed warnings, the average speed in each bin, and the number of accelerator releases and brake pedal presses were also similarly counted.

We also investigated gaze and speed behaviour at speed limit transitions. Speedometer glance rates and average speed were calculated as with the speed warnings, but the speed limit transition points were used instead of the warnings. Participants in the control group did not receive any speed warnings, but their data was still included in the speed limit transition analysis.

\section{Results}

\subsection{Speeding}

We first tested the effect of the speed warning system on speed compliance. The effect of the speed app (with speed

Table 1 Descriptive statistics of the three groups of drivers

\begin{tabular}{llll}
\hline & Active $(\boldsymbol{n}=5)^{\mathbf{a}}$ & Passive $(\boldsymbol{n}=6)$ & ${\text { Control }(\boldsymbol{n}=6)^{\mathbf{a}}}^{\mathbf{a}}$ \\
\hline Age in years (mean, SD) & $28.80(6.72)$ & $26.00(5.70)$ & $23.00(4.36)$ \\
Male $(\mathrm{n}, \%)$ & $2(40)$ & $5(83.3)$ & $2(33.3)$ \\
Years licensed driver & $10.00(6.59)$ & $6.00(5.73)$ & $9.83(4.79)$ \\
Km driven per week (mean, SD) & $80.00(34.64)$ & $143.83(181.30)$ & $196.67(168.13)$ \\
\hline
\end{tabular}

${ }^{\mathrm{a}}$ Two participants, one from the active group and one from the control group, were excluded from the analysis due to bad quality eye-tracking data 
app vs. control) and the speed limit $(100,80,60 \mathrm{~km} / \mathrm{h})$ on the proportion of time spent speeding over $3 \mathrm{~km} / \mathrm{h}$ the speed limit was tested with mixed ANOVA. Speeds from the first and last sections without warnings were excluded for this analysis, as well as the short $30 \mathrm{~km} / \mathrm{h}$ roadworks section.

Both main effects were present $(p<.05)$, but most importantly, also the interaction of the group and the speed limit was statistically significant, F $(1.178,17.67)=18.51$, $p<.001, \eta_{\mathrm{p}}^{2}=0.55$, Greenhouse-Geisser corrected for sphericity, $\varepsilon=0.589$. The control group exceeded the speed limit most often, especially when driving in the $60 \mathrm{~km} / \mathrm{h}$ zone (Fig. 3). Having a speed advisory system reduced the proportion of time spent speeding in the $60 \mathrm{~km} / \mathrm{h}$ zone $(p<.001)$ and the $80 \mathrm{~km} / \mathrm{h}$ zones $(p<.05)$ corrected for multiple comparisons with Tukey method). In the $100 \mathrm{~km} /$ $\mathrm{h}$ zones the difference was in the same direction, but not statistically significant $(p>.05)$.

\subsection{Glances to the speedometer and speed app}

Speedometer and speed app glance durations were rather short (speedometer: $\mathrm{Mdn}=310 \mathrm{~ms}, \mathrm{M}=348 \mathrm{~ms}, \mathrm{SD}=173$, range $=70-2040 \mathrm{~ms}$; speed app: $M d n=255 \mathrm{~ms}, M=230$ $\mathrm{ms}, \mathrm{SD}=132$, range $=80-1210 \mathrm{~ms})$. Speedometer glance durations recorded in the simulator were also shorter than those recorded on a motorway at $90 \mathrm{~km} / \mathrm{h}$ with an instrumented car $(\mathrm{Mdn}=580 \mathrm{~ms}, \quad \mathrm{M}=616 \mathrm{~ms}, \quad \mathrm{SD}=238$, range $=100-2350 \mathrm{~ms})$. Glance rates to the speed app were very low. The participants glanced at the speed app typically only when setting a new speed limit in the active condition (Supplementary Table 2).
Speedometer glance rates showed a dependency on the speed limits (Fig. 4). Therefore, a mixed ANOVA was used to analyse the effect of the group (with speed app vs. control) and the speed limit $(100,80,60 \mathrm{~km} / \mathrm{h})$. As before, baseline sections and $30 \mathrm{~km} / \mathrm{h}$ roadworks section were excluded. The main effect of speed limit was significant, $\mathrm{F}(2,30)=22.10, p<.001, \eta_{\mathrm{p}}^{2}=0.60$. Post hoc comparison showed that the glance rate was higher in the $60 \mathrm{~km} / \mathrm{h}$ zone compared to 80 and $100 \mathrm{~km} / \mathrm{h}$ zones $(p<.001$, corrected for multiple comparisons with Tukey method). However, there was no significant main effect of group or interaction between group and speed limit $(p>.05)$.

In the simulator, the speedometer glance rates were up to three times larger than on the motorway experiment. On a motorway, the more experienced participants had a speedometer glance rate $\mathrm{M}=0.07$ ( $\mathrm{SD}=$ $0.04)$, and less experienced $\mathrm{M}=0.16(\mathrm{SD}=0.07)$. On the motorway, the average speed was $87 \mathrm{~km} / \mathrm{h}$. Our control group in the simulator had speedometer glance rate $\mathrm{M}=0.24(\mathrm{SD}=0.06)$ at $100 \mathrm{~km} / \mathrm{h}$ sections with an average speed of $95 \mathrm{~km} / \mathrm{h}$.

The speedometer glance rates were calculated relative to the accelerator releases outside baselines to investigate if drivers preferred to look at the speedometer before making a release (Fig. 5). Glances showed a preference to look before releasing. The speedometer glance rate $0-1 \mathrm{~s}$ before was compared to the overall speedometer glance rate. The speedometer glance rate was $\mathrm{Mdn}=0.15$ glances $/ \mathrm{s}$ higher $(95 \%$ CI $[0.11,0.20]$ just

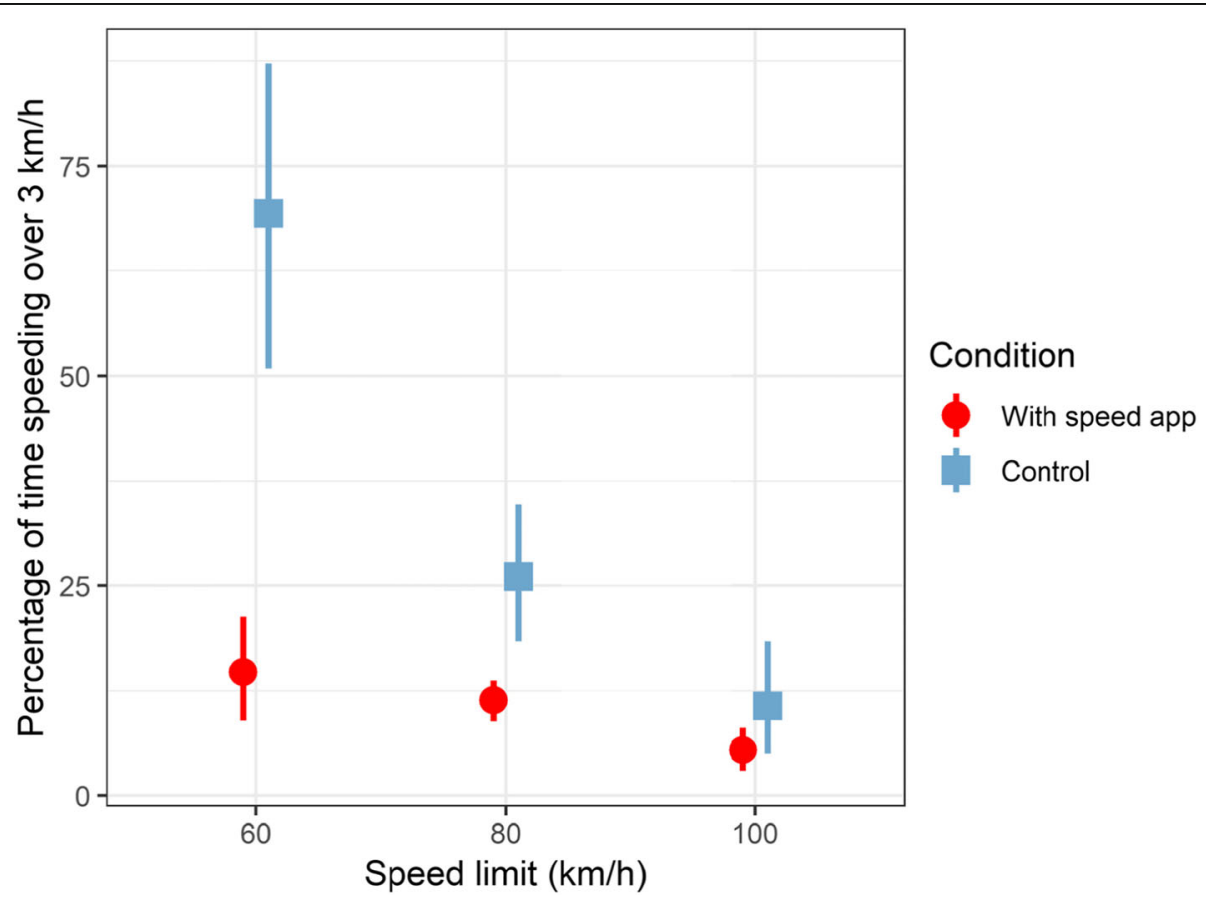

Fig. 3 Speeding as a function of speed limit and condition (with speed app vs. control) 


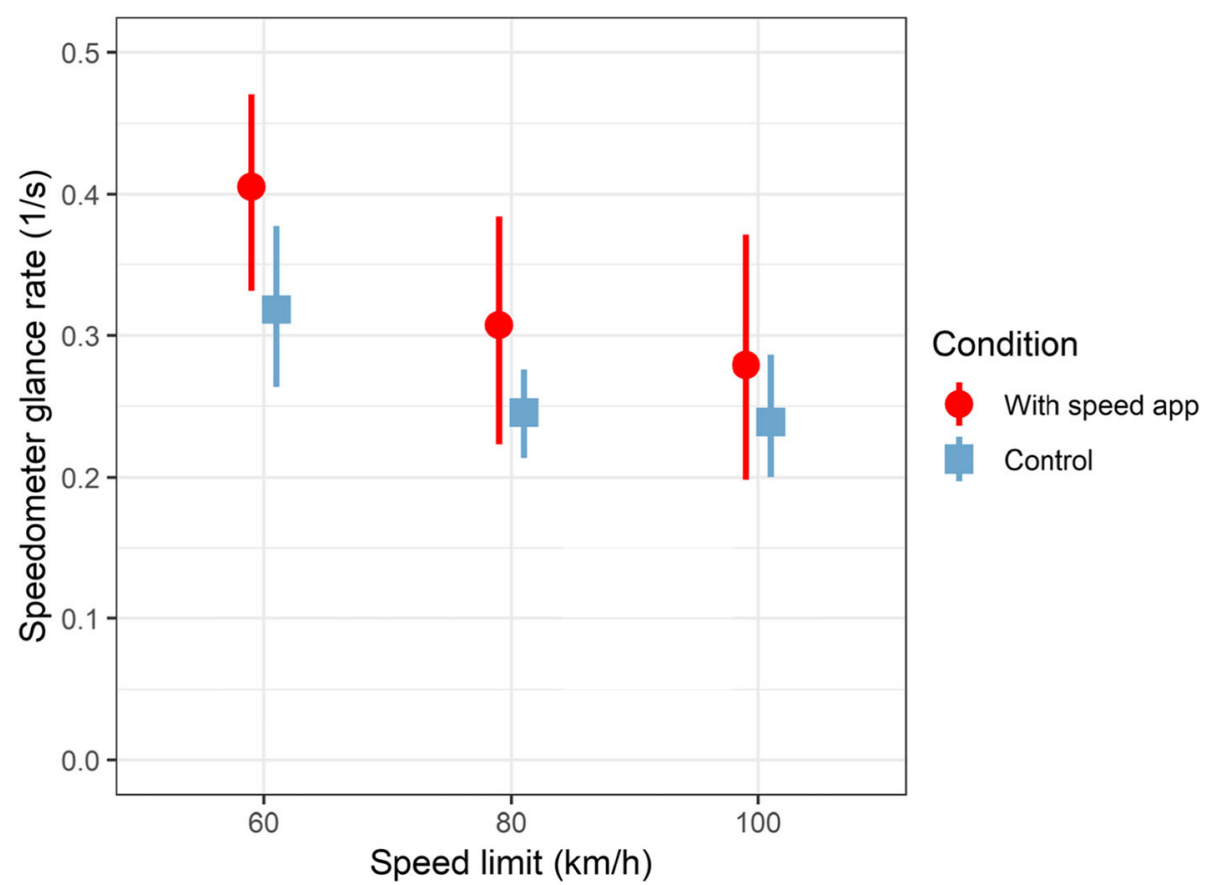

Fig. 4 Speedometer glance rate as a function of the speed limit and group (with speed app vs. control). Means and 95\% Cl

before compared to the overall rate (Paired Wilcox signed-rank test, $p<.01)$. Visually, the increase appears to be more pronounced when driving with the speed app than without, but the difference was not statistically significantly larger (Paired Wilcox signedrank test, n.s).

\subsection{Speed warnings}

The number of speed warnings received varied between drivers from 0 to 21, but on average, the speed warnings were rare (Table 2). Due to the limited number of speed warnings, it was not feasible to analyse in detail how for example the vertical curvature of the road may have

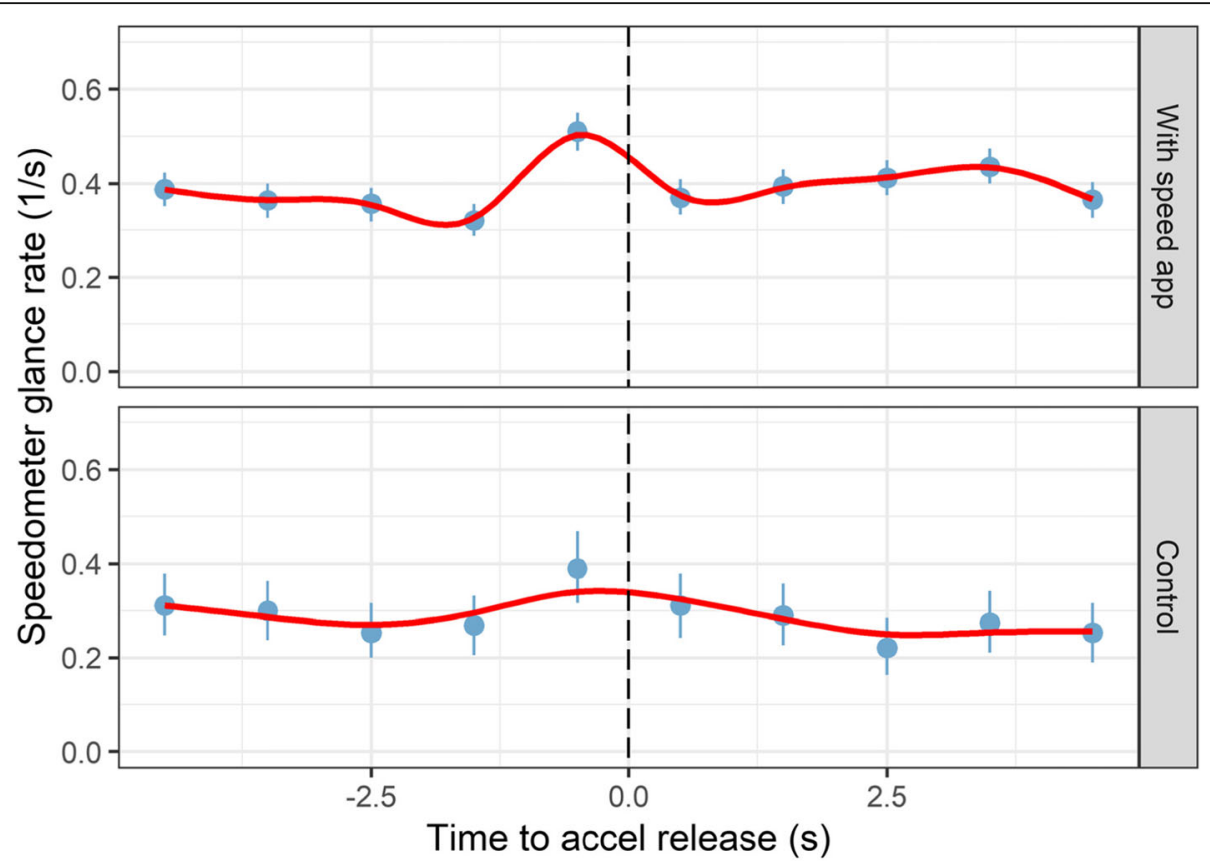

Fig. 5 The average rate of speedometer glances with 95\% Cl in one-second bins relative to accelerator releases by the group (rows). The two conditions with the speed app combined 
Table $\mathbf{2}$ The number of speed warnings by the condition

\begin{tabular}{llllll}
\hline Condition & Min & Max & Mdn & M & SD \\
\hline Active & 0 & 21 & 5.0 & 7.40 & 8.14 \\
Passive & 2 & 10 & 7.5 & 6.83 & 3.37 \\
\hline
\end{tabular}

influenced the speed warnings. Nevertheless, a histogram representing the distribution of the warnings along the road (Fig. 6) suggested there were some locations on the road, where warnings were more frequent. The first such location occurred after $9.7 \mathrm{~km}$ of driving where the 100 $\mathrm{km} / \mathrm{h}$ speed limit changed to $80 \mathrm{~km} / \mathrm{h}$. Here the road also had a slight downhill gradient (less than $.02 \%$ ), requiring more active speed control. The following speed transition, from $80 \mathrm{~km} / \mathrm{h}$ to $60 \mathrm{~km} / \mathrm{h}$ at $15.7 \mathrm{~km}$, also seemed to trigger many warnings. The $60 \mathrm{~km} / \mathrm{h}$ sign was at the end of a horizontal curve, just before an intersection partially occluded by a building. The last concentration of speed warnings occurred at the $100 \mathrm{~km} / \mathrm{h}$ zone, where the drivers were driving behind a truck and had the opportunity to overtake it on a passing lane.

\subsection{Speedometer glance rates relative to the speed warnings}

We hypothesised that receiving a speed warning would increase the speedometer glance rate, as drivers need to adapt their speed. We were also interested to see if speeding could be related to lapses of cognitive control, visible as fewer speedometer glances before a warning. Speedometer glance rates relative to the speed warnings events confirm these expectations (Fig. 7). Speedometer glance rate peaked $2-3 \mathrm{~s}$ after a warning and was at lowest 2-6 s seconds before. Drivers also sometimes could anticipate warnings, as speedometer glance rate started to increase again $0-2 \mathrm{~s}$ before a warning was triggered.

The average speed profile at warnings showed that glance behaviour was related to speed adaptation (Fig. 8). Speed started to increase $10 \mathrm{~s}$ before a warning, reaching its highest value $0-2 \mathrm{~s}$ before the warning. It took on average $5 \mathrm{~s}$ to return to the speed level preceding the speeding event. The accelerator release and brake pedal press rates showed that speed adaptation was indeed due to drivers' actions (Fig. 9). The accelerator release rates $0-2 \mathrm{~s}$ before were at a similar level compared to $0-2 \mathrm{~s}$ after (before: $\mathrm{M}=0.23, \mathrm{SD}=0.07$, after: $\mathrm{M}=0.19, \mathrm{SD}=0.14$, Paired Wilcox signed-rank test n.s.), and the brake pedal press rate had already started to increase before the warning. This means that many drivers had started to slow down before a warning was triggered, at the same time as the speedometer glance rate began to increase.

The speedometer glance rate changes around the warnings were tested for statistical significance by comparing $5 \mathrm{~s}$ time intervals before and after warnings. The After interval was set $0-5 \mathrm{~s}$ after a warning, because the speed profile indicated that it took approximately $5 \mathrm{~s}$ to slow down after a warning was triggered. The Before interval was defined to be $2-7 \mathrm{~s}$ prior to a warning $(<2 \mathrm{~s}$ before the warning was excluded, because many drivers began adapting their speed in anticipation of the warning). For each participant, average speedometer glance rates were calculated for these time intervals. Paired Wilcox signed-rank tests were used for testing.

Speedometer glance rates were $\mathrm{Mdn}=0.23$ higher in the After interval compared to the Before interval (95\% CI $[0.15,0.33], \mathrm{V}=55, p=.002)$. The intervals were also compared to the overall speedometer glance rate on the test sections. Speedometer glance rate in the Before interval was $\mathrm{Mdn}=-0.10$ lower than the overall rate

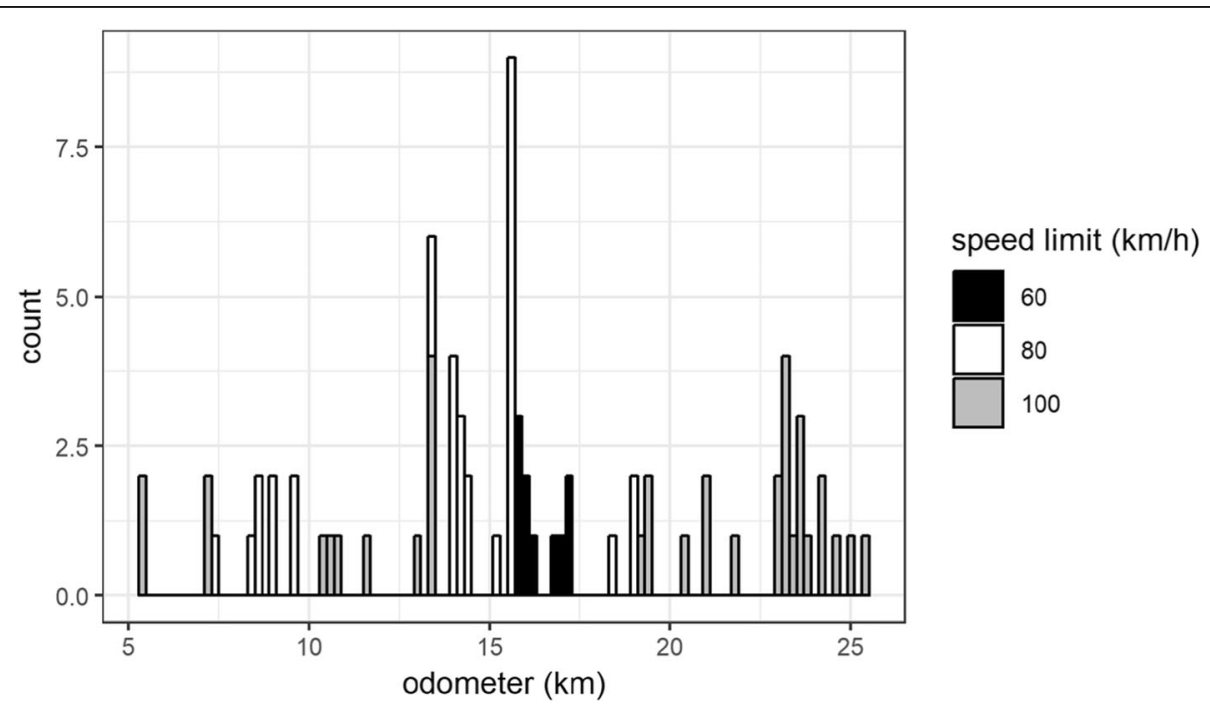

Fig. 6 The number of speed warnings along the road in $200 \mathrm{~m}$ bins. Odometer reading along the road $(\mathrm{km})$ on the $\mathrm{x}$-axis 


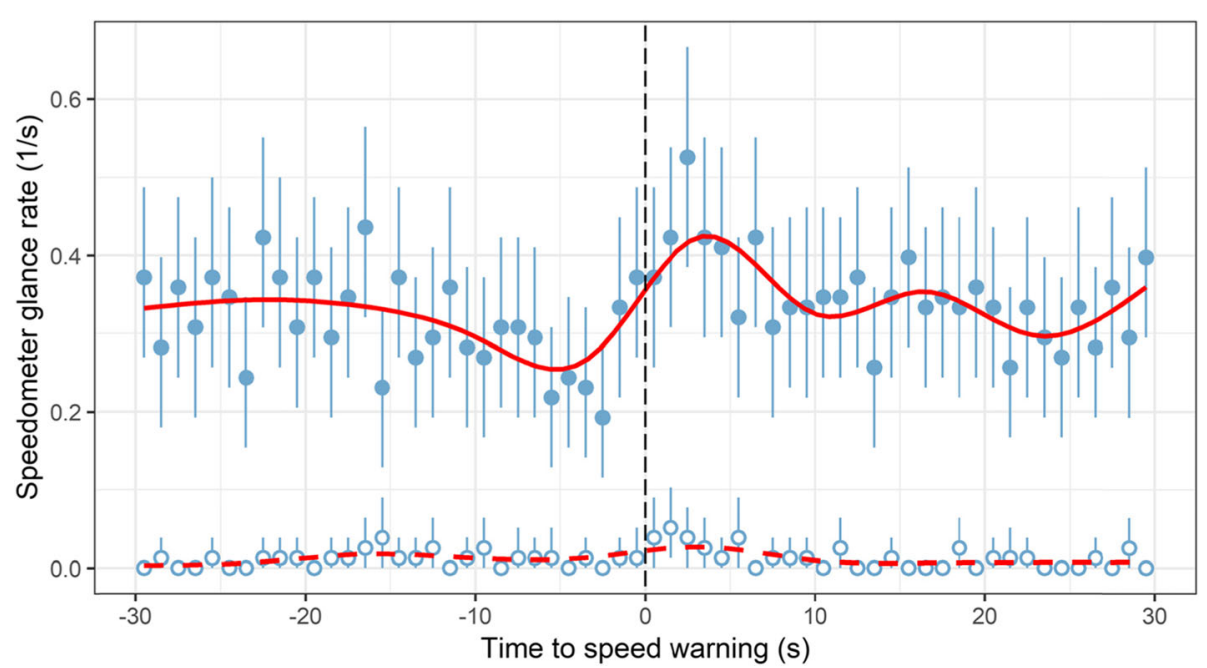

Fig. 7 Speedometer glance rates before and after speed warnings, calculated in one-second bins over all the speed warnings. Mean and $95 \% \mathrm{Cl}$ shown with points and ranges, with loess smoothed solid red line. For comparison, speed app glance rates similarly on the bottom (the circles with ranges, with loess smoothed dashed red line)

(95\% CI $[-0.22,-0.01], \mathrm{V}=7, p=.037)$. In the After interval, the speedometer glance rate was $\mathrm{Mdn}=0.13$ higher $(95 \%$ CI $[0.03,0.23], \mathrm{V}=49, p=.027)$.

We wanted to also investigate if the speedometer glances preceded the accelerator releases also when done in response to a warning within $0-3 \mathrm{~s}$. Drivers could rely on the warning and make the decision to release the accelerator without looking at the speedometer. Figure 10 shows that there was still a tendency to increase looking at the speedometer before performing a release.

\subsection{Speed limit transitions}

We also analysed speed limit transitions, because we wanted to see if we could replicate the increase of speedometer glances during the speed adaption in another sort of speed adaptation situation. Speed profiles show that speed adaptation started 10 to $5 \mathrm{~s}$ before the transition, and lasted 5 to $10 \mathrm{~s}$ after it (Fig. 11). Speedometer glance rate showed increases during the same period when the drivers adapted their speed to the new speed limit (Fig. 12).

\section{Discussion}

The aim of the study was to examine the influence of a speed warning system on drivers' speedometer monitoring, and how drivers respond when they receive a warning. We wanted to see if the presence of a speed warning system would increase the speedometer monitoring, measured as the speedometer glance rate, as would be expected if the system increased drivers' cognitive control over their speed $[7,22]$. In addition to such

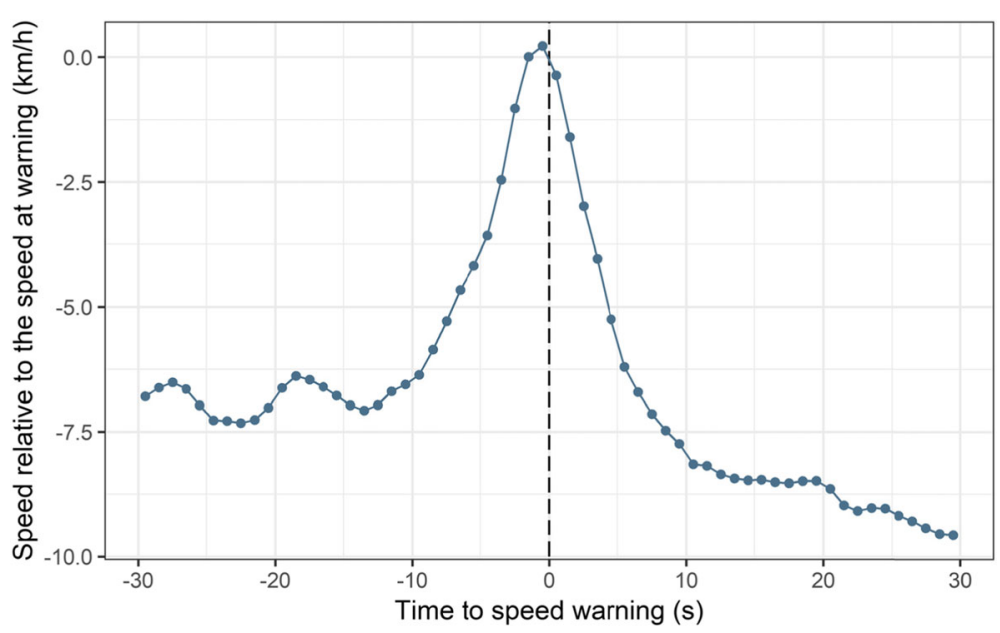

Fig. 8 Speed profile at warnings: the average speed $30 \mathrm{~s}$ before and after the warning relative to the speed at the warning 


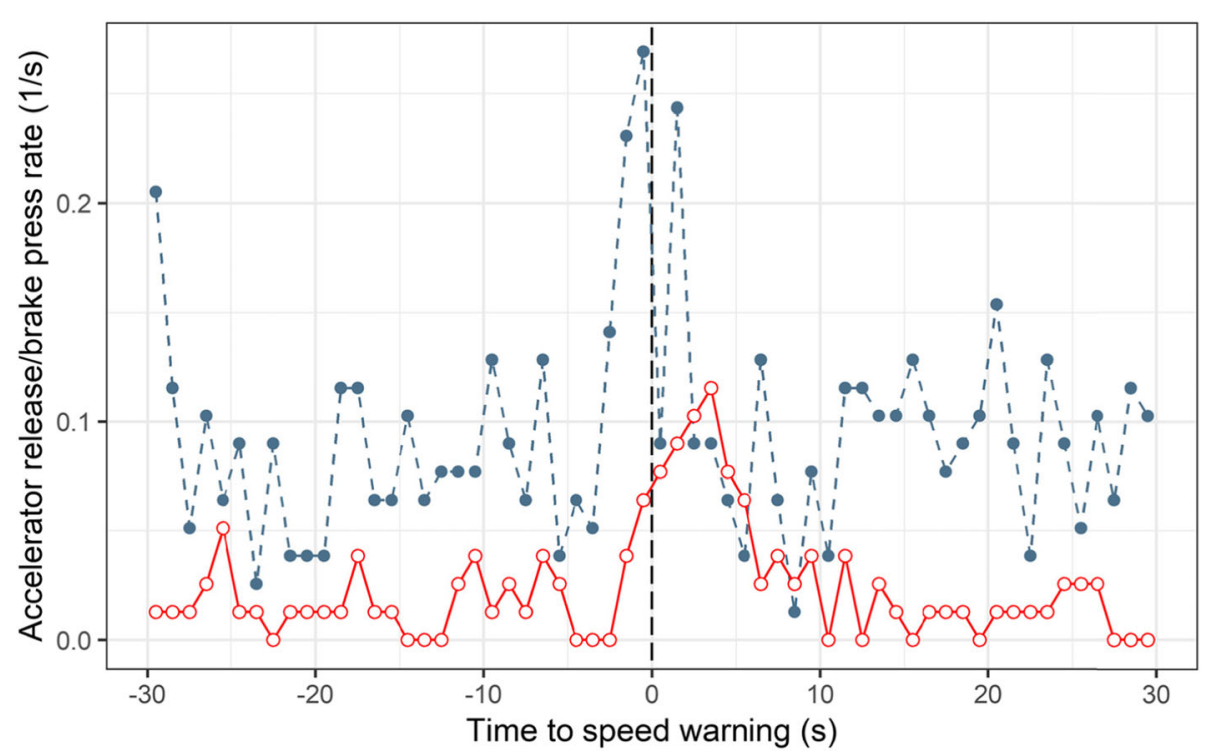

Fig. 9 The average rate of accelerator pedal releases (blue points, connected with dashed line) and brake pedal presses (red circles, connected with solid line) relative to the speed warnings

overall effect, we expected to see an increase of speedometer glances after receiving a warning, and possibly decrease of speedometer glances before, if speeding were linked to a momentary lapse of cognitive control over speed.

Drivers frequently glanced at the speedometer, but the speedometer glance rates were not different with or without a speed app. Especially, in the $60 \mathrm{~km} / \mathrm{h}$ speed limit, where the control group exceeded the speed limit most often and where there was most speeding by the control group, the speedometer glance rates were not significantly higher with the system compared to the control.

Drivers often looked at the speedometer just before they released an accelerator. This pattern appeared to be more pronounced with a speed warning system, but not statistically significantly so. In other words, there is no indication the warning system would have changed the speedometer monitoring in general. It is also good to note that there was no indication that a speed warning system would lead drivers to complacency [19], where

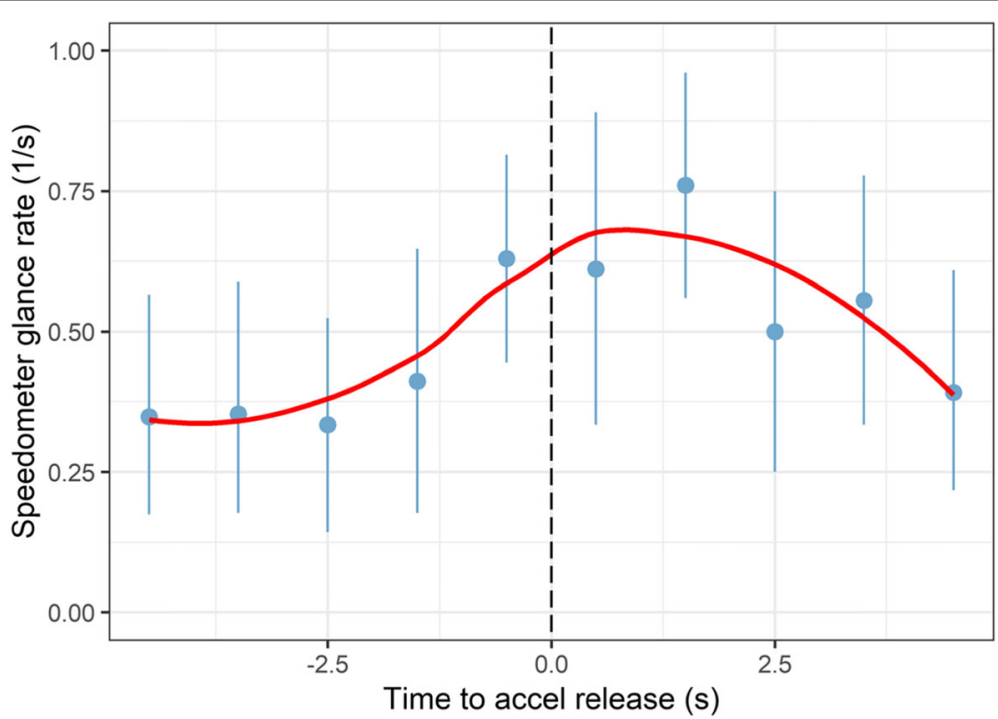

Fig. 10 Speedometer glance rate relative to the accelerator releases 0-3 after a warning. Mean and 95\% Cl shown, with loess smoothed solid red line 


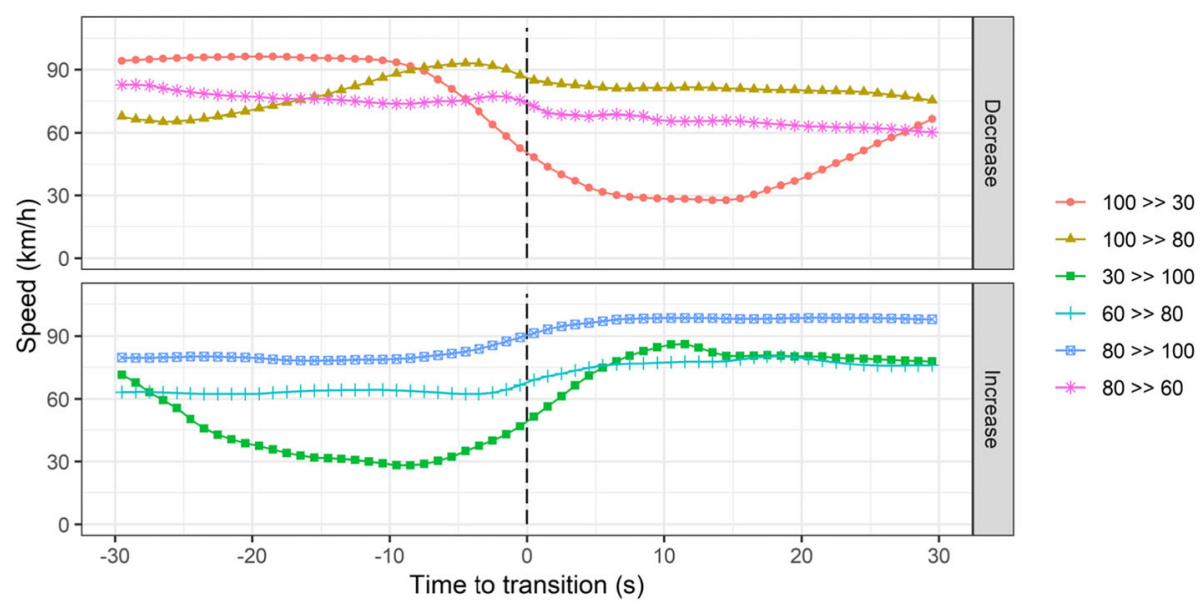

Fig. 11 Average speed profiles for each speed limit transition $30 \mathrm{~s}$ before and after the speed limit sign. Separate figures for the speed limit decreases and increases

they would reduce their speedometer monitoring, waiting for the warnings instead.

The speed limit, however, influenced the speedometer glance rates among all groups. The speedometer glance rate was higher in the $60 \mathrm{~km} / \mathrm{h}$ zone compared to 80 $\mathrm{km} / \mathrm{h}$ and $100 \mathrm{~km} / \mathrm{h}$ zones. In the experiment, the 60 $\mathrm{km} / \mathrm{h}$ segment had a similar road geometry to $100 \mathrm{~km} / \mathrm{h}$ and $80 \mathrm{~km} / \mathrm{h}$ segments. The higher speedometer glance rate can be interpreted to reflect increased cognitive control over the speed when the visual cues indicated a higher optimal speed than the speed limit would allow. The situation is analogous to asking drivers to drive slower on the same road. Recarte and Nunes [22] reported that time spent looking at the speedometer

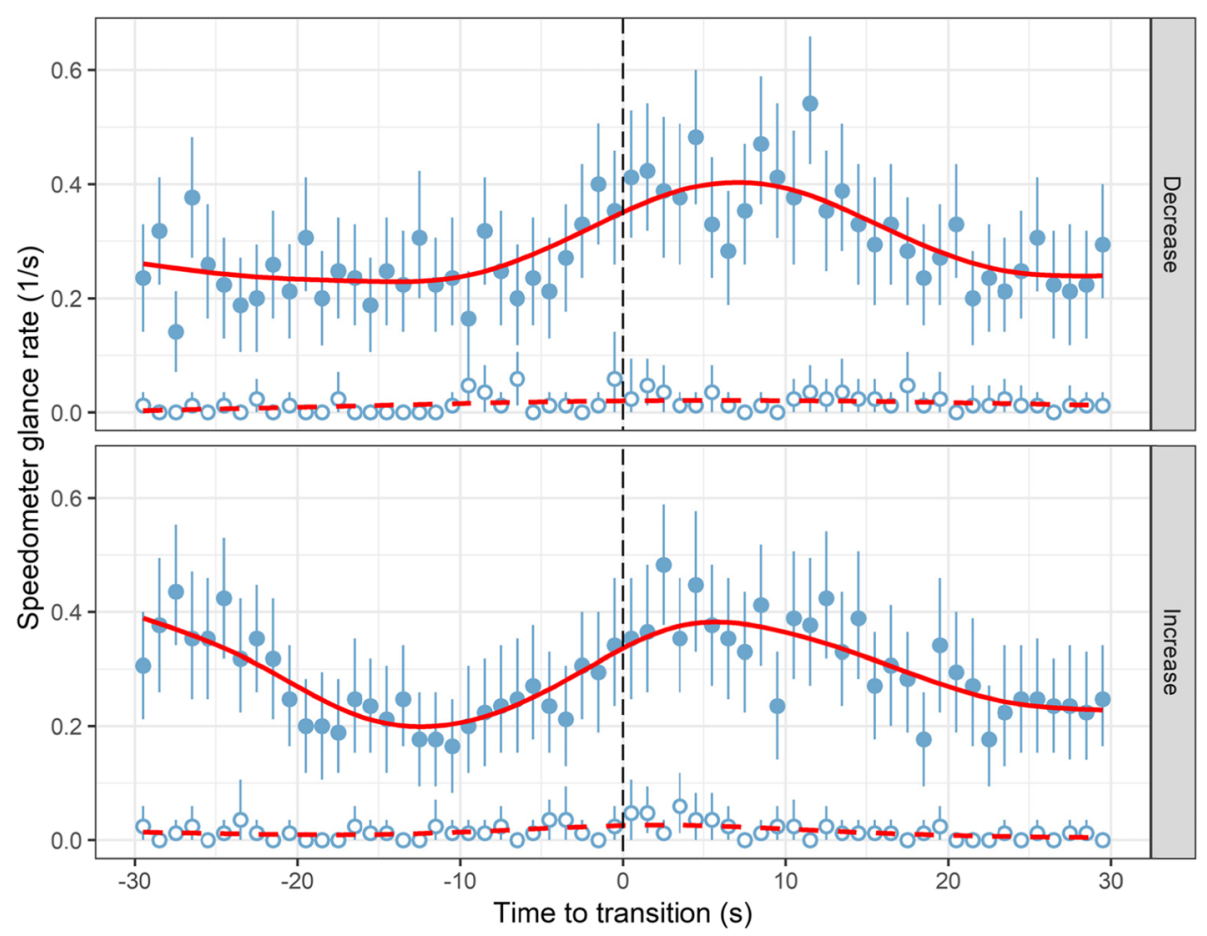

Fig. 12 Speedometer glance rate glances relative to the speed limit transitions. Mean and 95\% Cl shown with points and ranges, with loess smoothed solid red line. For comparison, speed app glance rates on the bottom of the figures for comparison (the circles with ranges, with loess smoothed dashed red line). Separate figures for the speed limit decreases and increases. Glance rates are averages over all the participants and transitions 
increased three-fold when drivers were asked to drive between 90 and $100 \mathrm{~km} / \mathrm{h}$ compared to free speed choice on a motorway with $120 \mathrm{~km} / \mathrm{h}$ speed limit.

The time course of speedometer glances relative to the speed warnings indicated that speedometer glance rates decreased $2-7 \mathrm{~s}$ before the warnings and started to increase $2 \mathrm{~s}$ seconds prior to the warning. Speed started to increase approximately $10 \mathrm{~s}$ before a warning was triggered. Within $0-2 \mathrm{~s}$ before the warning, the average speed was higher than $0-1 \mathrm{~s}$ after the warnings, suggesting that drivers often noticed before the warning that they are going over the speed limit, and therefore glanced at the speedometer and started to reduce their speed, typically by releasing the accelerator. The decrease of speedometer glances prior warnings suggest that a partial explanation for speeding can be a momentary lapse of cognitive control over speeds (cf. [7]).

The results also demonstrated that drivers do not only react to speed warnings, but they anticipate them and start speed adaptation before a warning occurs. This suggests that part of the speed reducing the effect of a speed warning system can be that drivers recognize when they are going over the speed limit and try to react by reducing their speed.

The analysis of speedometer glances during the speed limit transitions supported the interpretation that the increase in the speedometer glance rate with warnings is related to speed adaptation. Speedometer glance rate started to increase when the average speed profile started to change, and lasted until the transition to the new speed level was finished. Noteworthy, there was no difference between speed transition upwards or downwards.

Regarding the visual distraction of ISA, Starkey et al. [23] already concluded that the speed warning system used in the experiment is unlikely to visually distract the driver, as the number of glances to the speed app was very small, and most of them were with the active system, which required the driver to manually set the current speed limit. In this sense, the speed warnings systems appear to be very different from constantly updating visual eco-driving feedback systems that can attract a significant portion of drivers' visual attention [12]. The further analysis of speedometer monitoring does not suggest that a speed warning system would be very distracting, either. Glances to the speedometer are frequent, but because most of the glances to the speedometer are short, less than $1 \mathrm{~s}$ [9] and thus clearly under the recommended $2 \mathrm{~s}$ threshold for off-road glances, they are unlikely to distract the driver [18]. However, to minimize any potential distraction, it could be beneficial to avoid giving speed warnings in situations which may require fast response from the driver, e.g. when following a lead vehicle at short distance or when overtaking, because those may trigger drivers to look at the speedometer.

The current results suggest speed warning systems can be especially effective to counteract unintentional speeding caused by inattention over the control of speed. Systems can be also more effective if the drivers are able to predict when a warning would be triggered, enabling them to use available perceptual cues to re-engage their cognitive control even without a warning. A better understanding of the temporal coupling between speedometer glances, speed regulation and speed warnings could be used to design ISA systems adapt to the driver's speed regulation strategies, for example by not issuing a warning if the driver has just glanced the speedometer and it is likely that he/she will release the accelerator soon. Such adaptive designs may increase the user acceptance of ISA systems and help to realize their true potential to improve safety.

\subsection{Limitations and future studies}

The experimental settings have likely affected driver behaviour. Speedometer glance rates in the simulator were up to three times higher than those calculated from data collected on a motorway, without a speed warning system but with an instruction to keep the speed at $90 \mathrm{~km} / \mathrm{h} \mathrm{[16].}$ The participants were aware that the study was about speed warning systems and had experimenters monitoring their driving, which may have led them to monitor their speed more closely than they usually would. Also, the simulator may not replicate all the perceptual cues which can be used for the control of speed, requiring the drivers to exert more cognitive control over the speed with the help of speedometer glances, further increasing their speedometer glance rates. Inflated speedometer glances rates may have masked differences between the control and speed warning groups. The present study was also limited in the number of participants.

The current results cannot be generalised to intentional speeding, because we would assume that most of the participants tried to comply with the presented warnings. It would be valuable to check if the observed synchronization of glances and speed warnings would emerge also using naturalistic data with a commercially available ISA system, and if glance patterns could be used to distinguish intentional and unintentional speeding events.

\section{Conclusion}

Speedometer glances can be used as an indicator of cognitive control over driving speed. The current results suggest that before speed warnings the speedometer glance rate may decrease, and after warnings it increases. This indicated the speed warnings re-engage the drivers to cognitive control of speed, which they may have momentarily lost before speeding. Drivers anticipate warnings by increasing their speedometer glance rate and adjusting their speed a couple of seconds before a warning is triggered. The present study did not find evidence that a speed warning system would change the speedometer monitoring in general. The results suggest that warnings can 
effectively counteract unintentional speeding, caused by momentary inattention over the speed control. Further research linking speeding to distractive events and/or mindwandering could provide direct support for this idea.

\section{Supplementary information}

Supplementary information accompanies this paper at https://doi.org/10. 1186/s12544-020-00408-8.

Additional file 1: Supplementary Table 1. Speed behaviour by the experimental group and road sections. Mean and standard deviation.

Additional file 2: Supplementary Table 2. Speedometer and speed app glance rates by the experimental group and speed limit. Mean and standard deviation.

\section{Acknowledgements}

The authors would like to express appreciation for the support of the funder, the NZ Transport Agency [Project TAR 16/18] and members of the project's steering group. The current study was a part of a larger study performed in the project.

\section{Authors' contributions}

NS and SC conceived the study; EL, NM, NS and SC designed the study and acquired the data; EL analysed results and wrote the first draft; EL, NM, NS and SC revised the manuscript. All authors read and approved the final manuscript.

\section{Funding}

The study received funding from NZ Transport Agency [Project TAR 16/18]. The funder had no role in the design, collection, analysis, or interpretation of data and in writing the manuscript.

\section{Availability of data and materials}

The datasets used and/or analysed during the current study are available from the corresponding author on reasonable request.

\section{Competing interests}

The authors declare that they have no competing interests.

\section{Author details}

${ }^{1}$ VTT Technical Research Centre of Finland, Espoo, Finland. ${ }^{2}$ National Youth Sport Institute, Singapore, Singapore. ${ }^{3}$ Transport Research Group, School of Psychology, University of Waikato, Hamilton, New Zealand.

\section{Received: 26 September 2019 Accepted: 8 March 2020}

\section{0.0 .}

\section{References}

1. Aarts, L., \& Van Schagen, I. (2006). Driving speed and the risk of road crashes: A review. Accident Analysis and Prevention, 38(2), 215-224. https:// doi.org/10.1016/j.aap.2005.07.004

2. Adell, E., Várhelyi, A., \& Hjälmdahl, M. (2008). Auditory and haptic systems for in-car speed management - A comparative real life study. Transportation Research Part F: Traffic Psychology and Behaviour, 11(6), 445-458. https://doi. org/10.1016/j.trf.2008.04.003.

3. Ahie, L. M. Charlton, S. G. \& Starkey, N. J. (2015). The role of preference in speed choice. Transportation Research Part F: Traffic Psychology and Behaviour, 30, 66-73. https://doi.org/10.1016/j.trf.2015.02.007.

4. Charlton, S. G., \& Starkey, N. J. (2016). Risk in our midst: Centrelines, perceived risk, and speed choice. Accident Analysis and Prevention, 95, 192-201. https:// doi.org/10.1016/j.aap.2016.07.019.

5. Charlton, S. G., Starkey, N. J., \& Malhotra, N. (2018). Using road markings as a continuous cue for speed choice. Accident Analysis and Prevention, 117, 288-297. https://doi.org/10.1016/j.aap.2018.04.029

6. Elvik, R. (2013). A re-parameterisation of the power model of the relationship between the speed of traffic and the number of accidents and accident victims. Accident Analysis and Prevention, 50, 854-860. https://doi. org/10.1016/j.aap.2012.07.012
7. Engström, J., Markkula, G., Victor, T., \& Merat, N. (2017). Effects of cognitive load on driving performance: The cognitive control hypothesis. Human Factors, 59(5), 734-764. https://doi.org/10.1177/ 0018720817690639

8. European Commission. (2019). Proposal for a Directive of the European Parliament and of the Council amending Directive 2008/96/EC on road infrastructure safety management COM/2018/274 final - 2018/0129 (COD). Retrieved from https:/eurlex.europa.eu/legal-content/EN/TXT/?uri=CELEX:52018PC0274

9. Green, P. (1999). Visual and task demands of driver information systems. In UMTRI 98-16 Retrieved from http://deepblue.lib.umich.edu/bitstream/202 7.42/1269/2/92250.0001.001.pdf.

10. Harms, L. (1991). Variation in drivers' cognitive load. Effects of driving through village areas and rural junctions. Ergonomics, 34(2), 151-160. https://doi.org/10.1080/00140139108967303.

11. Hayhoe, M. M., Shrivastava, A., Mruczek, R., \& Pelz, J. B. (2003). Visual memory and motor planning in a natural task. Journal of Vision, 3(1), 49-63. https:// doi.org/10.1167/3.1.6.

12. Jamson, S. L., Hibberd, D. L., \& Jamson, A. H. (2014). Drivers' ability to learn eco-driving skills; Effects on fuel efficient and safe driving behaviour. Transportation Research Part C: Emerging Technologies, 58, 657-668. https:// doi.org/10.1016/j.trc.2015.02.004.

13. Land, M., Mennie, N., \& Rusted, J. (1999). The roles of vision and eye movements in the control of activities of daily living. Perception, 28(11), 1311-1328. https://doi.org/10.1068/p2935.

14. Lehtonen, E., Lappi, O., Koirikivi, I., \& Summala, H. (2014). Effect of driving experience on anticipatory look-ahead fixations in real curve driving. Accident Analysis and Prevention, 70, 195-208. https://doi.org/10.1016/j.aap. 2014.04.002

15. Lehtonen, E., Lappi, O., \& Summala, H. (2012). Anticipatory eye movements when approaching a curve on a rural road depend on working memory load. Transportation Research Part F: Traffic Psychology and Behaviour, 15(3). https://doi.org/10.1016/j.trf.2011.08.007.

16. Lehtonen, E, Lappi, O., Koskiahde, N., Mansikka, T, Hietamäki, J, \& Summala, H. (2018). Gaze doesn't always lead steering. Accident Analysis \& Prevention, 121(August), 268-278. https://doi.org/10.1016/j.aap.2018.09.026.

17. Lehtonen, E., Lappi, O., Kotkanen, H., \& Summala, H. (2013). Look-ahead fixations in curve driving. Ergonomics, 56(1), 34-44. https://doi.org/10.1080/ 00140139.2012.739205

18. NHTSA. (2013). Visual-manual NHTSA driver distraction guidelines for invehicle electronic devices. Federal Register, 78(81), 24818-24890 Retrieved from https://www.federalregister.gov/d/2013-09883.

19. Parasuraman, R., \& Manzey, D. H. (2010). Complacency and bias in human use of automation: An attentional integration. Human Factors, 52(3), 381-410. https://doi.org/10.1177/0018720810376055.

20. Patla, A. E., \& Vickers, J. N. (1997). Where and when do we look as we approach and step over an obstacle in the travel path? Neuroreport, 8(17) 3661-3665 Retrieved from http://www.ncbi.nlm.nih.gov/pubmed/9427347

21. Pelz, J. B., \& Canosa, R. (2001). Oculomotor behavior and perceptual strategies in complex tasks. Vision Research, 41(25-26), 3587-3596. https:// doi.org/10.1016/S0042-6989(01)00245-0.

22. Recarte, M. A., \& Nunes, L. (2002). Mental load and loss of control over speed in real driving. Towards a theory of attentional speed control. Transportation Research Part F: Traffic Psychology and Behaviour, 5(2), 111-122. https://doi.org/ 10.1016/S1369-8478(02)00010-4.

23. Starkey, N. J., Charlton, S. G., Malhotra, N., \& Lehtonen, E. (2020). Drivers' response to speed warnings provided by a smart phone app. Transportation Research Part C: Emerging Technologies, 110(October 2019), 209-221. https:// doi.org/10.1016/j.trc.2019.11.020.

24. Warner, H. W., \& Åberg, L. (2008). The long-term effects of an ISA speed-warning device on drivers' speeding behaviour. Transportation Research Part F: Traffic Psychology and Behaviour, 11(2), 96-107. https://doi.org/10.1016/j.trf.2007.08.002.

25. Warner, H. W., Özkan, T., \& Lajunen, T. (2010). Drivers' propensity to have different types of intelligent speed adaptation installed in their cars. Transportation Research Part F: Traffic Psychology and Behaviour, 13(3), 206-214. https://doi.org/10.1016/.jtrf.2010.04.005.

\section{Publisher's Note}

Springer Nature remains neutral with regard to jurisdictional claims in published maps and institutional affiliations. 